\title{
Mobilidade intrageracional de rendimentos no Brasil
}

\author{
MARIANGELA FURLAN ANTIGO \\ ANA FLÁVIA MACHADO*
}

This work investigates the earnings mobility in Brazil, considering the period before and after the observed fall of inequality in the country. We used microdata from Monthly Employment Survey (PME/IBGE), from 1992 to 2009. It is possible to analyze mobility in an intra generational context. The mobility contributes to decrease income inequality. Given the fall of inequality in the country, if mobility persists, Brazil may experience, in the long run, greater income convergence.

Keywords: mobility; earnings; income inequality.

JEL Classification: J31; J62.

\section{INTRODUÇÃO}

Nos últimos anos, a economia brasileira passou por várias modificações que afetaram a distribuição do rendimento. Plano de estabilização bem-sucedido, valorização do salário mínimo, criação de políticas de transferência de renda são alguns exemplos de políticas que desconcentraram a distribuição pessoal da renda. Nesse contexto, surgem questões referentes à mobilidade de rendimentos. Foi intensa a mobilidade de rendimentos? Algum grupo demográfico foi beneficiado? Se sim, onde se localizavam na distribuição do rendimento? O objetivo deste trabalho é, portanto, identificar padrões de mobilidade diferenciados (ou não), levando em conta os atributos individuais e do mercado de trabalho.

Barros et al. (2006) e Soares et al. (2007) retratam que as mudanças nos níveis de renda do trabalho possibilitaram a menor concentração na distribuição do rendimento do trabalho. Diante desses resultados favoráveis, colocam-se questões

\footnotetext{
* Universidade Federal de Minas Gerais. E-mail: maantigo@cedeplar.ufmg.br; Universidade Federal de Minas Gerais E-mail: afmachad@cedeplar.ufmg.br. Submetido: 4/fevereiro/2011; Aprovado: 24/janeiro/2012.
} 
pertinentes à mobilidade de rendimentos. Muitos estudos analisam a evolução da desigualdade de renda considerando diferentes medidas. Entretanto, as medidas usuais de desigualdade, ao considerarem apenas a distribuição cross-section dos rendimentos, tornam-se insuficientes, dado que expressam apenas uma análise estática e não um retrato da dinâmica dos padrões de mobilidade.

De acordo com Solon (2001), o aumento da desigualdade ao longo do tempo pode ser decorrente de um diferencial crescente nos rendimentos entre indivíduos mais pobres e mais ricos. Isto sustenta uma desigualdade crescente de longo prazo. Entre os fatores que justificam a desigualdade cross-section, o autor mostra que diferenças entre os atributos individuais de uma mesma coorte acarretam uma variação permanente dos rendimentos, afetando a desigualdade de longo prazo. Um maior nível de escolaridade, por exemplo, pode levar determinada coorte a auferir maiores rendimentos, o que altera a configuração de longo prazo. Somando-se a isso, comportamentos e decisões que geram segmentação e discriminação salarial podem assegurar um grau elevado de mobilidade.

Ainda, no âmbito intrageracional as condições de variáveis macroeconômicas refletem-se diretamente nos rendimentos individuais. O crescimento econômico, por exemplo, pode ocasionar uma mobilidade ascendente e períodos de estagnação, por sua vez, geram um comportamento oposto. Além disso, as políticas de transferência de renda, quando focalizadas na população mais pobre, podem influenciar a mobilidade.

Essas variáveis podem atingir os rendimentos de toda a sociedade ou subgrupos específicos. O Estado pode contribuir para este cenário por meio de políticas de capacitação, universalização do ensino, seguridade social, transferência de renda, políticas do mercado de trabalho como a valorização do salário mínimo, entre outras.

A mobilidade de rendimentos é, assim, um reflexo de decisões tomadas ex ante pelos indivíduos em investimento educacional, além de fatores independentes como condições macroeconômicas, fatores do mercado de trabalho e de cunho institucional que podem contribuir para o fenômeno. Caso a mobilidade afete apenas grupos específicos, isso pode significar menor desigualdade de longo prazo e, para uma dada desigualdade de renda, pode ser reflexo de um efeito compensatório resultante de uma mobilidade ascendente para menores rendimentos e/ou descendentes para maiores rendimentos. Dessa forma, a mobilidade de rendimentos completa a análise da desigualdade de renda, uma vez que a mobilidade pode alterar, consideravelmente, a distribuição ao longo do tempo.

Com a mobilidade podemos quantificar como a posição dos indivíduos no presente é (ou não) dependente de sua posição no período anterior e, assim, estabelecer uma relação com a desigualdade de renda. A mobilidade pode ser tratada no contexto inter e intrageracional. Considerando a primeira abordagem, os trabalhos a respeito da mobilidade se baseiam em correlações intergeracionais de rendimentos e padrão educacional entre pais e filhos e são motivados pela abordagem teórica de Becker e Tomes $(1979,1986)$. Esta questão começou a se desenvolver dentro da literatura econômica nos anos 1990, estimulada pela disponibilidade 
de dados em painéis internacionais e pela publicação de Solon $(1989,1992)$ que ressalta a importância de erros de medida e métodos para corrigi-los. Autores como Björklund e Jäntti (2000), Corak (2004), Solon (2002), Aydemir et al. (2005) e Erikson e Goldthorpe (2002) são alguns exemplos dentro da literatura que abordam esta questão. Um avanço nessa literatura é a mensuração da mobilidade na medida em que consideram, também, as elasticidades intergeracionais dos rendimentos entre as gerações como mostrado por Fields e Ok (1996), Solon (1999), Behrman et al. (2001) e Ermish e Nicoletti (2006). Entretanto, devido à limitação de dados organizados em painel para determinados países ou regiões, alguns autores como, por exemplo, Blanden et al. (2002) consideram a renda dos pais em detrimento dos rendimentos. Outros autores como Ermisch e Francesconi (2004), por sua vez, estimam a elasticidade intergeracional por meio de um escore de prestígio ocupacional - Hope-Goldthorpe score - de pais e filhos, partindo da constatação de que estes são fortemente relacionados aos rendimentos dos indivíduos.

Para o Brasil, a literatura relativa à mobilidade social intergeracional é vasta e autores como Pastore (1979), Valle e Silva (1973, 1992), Pastore e Haller (1993), Pastore e Valle e Silva (2000), Ferreira e Veloso (2004), Pero (2001, 2002), Andrade (1995, 2000), Jannuzzi (2000, 2001) e Pero e Szerman (2005) podem ser citados.

Embora existam muitos estudos que abordem a mobilidade, tanto sob a perspectiva sociológica quanto econômica, ainda existe uma lacuna a ser preenchida no que tange à mobilidade de rendimentos. Dois trabalhos podem ser destacados. O primeiro retrata a dinâmica da mobilidade de rendimentos do trabalho para o Brasil, realizado por Nascimento e Souza (2005) e baseado na Pesquisa Mensal de Emprego no período de 1984 a 2001. Inspirados por Buchinsky et al. (2003), os autores consideram os seguintes conceitos de mobilidade: movimento direcional e não direcional dos rendimentos, movimento de participação, dependência temporal e movimento posicional, não levando em consideração a mobilidade como equalizador de renda de longo prazo no Brasil, o que impede a inferência sobre a relação entre o comportamento da mobilidade e a desigualdade de renda no Brasil. O outro trabalho é de Figueiredo (2010) que, a partir de matrizes de transição markoviana com instrumentos dinâmicos propostos por Aebi et al. (1999) apud Figueiredo (2010) aplicados aos microdados da PNAD, no período de 1995 e 2005 , evidencia baixa mobilidade intrageracional.

Desse modo, este estudo pretende contribuir para a literatura sobre mobilidade intrageracional no Brasil, recorrendo a indicadores de mobilidade tradicionais e os desenvolvidos por Fields e Ok (1996). A contribuição deste trabalho é ressaltada pela singularidade do período de cobertura (1992 a 2009), quando mudanças importantes na estrutura de rendimentos no Brasil ocorreram, e porque faz uso de dados longitudinais, cuja natureza é bastante apropriada para o presente objetivo.

Recorremos à Pesquisa Mensal de Emprego (PME) do Instituto Brasileiro de Geografia e Estatística (IBGE). A PME se destaca por ser uma pesquisa organizada em forma longitudinal, ainda que sob formato de painel rotativo. As informações individuais reportadas na PME permitem mensurar a mobilidade em um contexto intrageracional. Contudo, esta pesquisa apresenta uma quebra temporal em 2002, 
quando o IBGE mudou a metodologia, em especial, o conceito de trabalho. Desse modo, a mobilidade é mensurada nos intervalos de 1992 a 2002 e 2002 a 2009 e nas seis regiões metropolitanas - Recife, Salvador, Belo Horizonte, São Paulo, Rio de Janeiro e Porto Alegre - que compõem a pesquisa. O painel rotativo permite acompanhar os domicílios durante quatro meses consecutivos e, após um intervalo de oito meses, eles voltam a ser investigados por mais quatro meses, quando são definitivamente excluídos da amostra. Este esquema de rotação garante o caráter longitudinal da pesquisa.

Assim, o artigo está dividido em quatro seções, considerando esta introdução. $\mathrm{Na}$ segunda, são relatados os indicadores de mobilidade e, na seção subsequente, os resultados obtidos. Por fim, discutimos os principais achados.

\section{INDICADORES DE MOBILIDADE}

Embora exista uma gama de trabalhos com o uso das matrizes de transição para captar a mobilidade dos rendimentos, trabalhos recentes têm mudado o enfoque de análise considerando alguns conceitos de mobilidade que, diferentemente das matrizes, possibilitam um estudo mais dinâmico e mais completo sobre os padrões fundamentais da mobilidade de rendimentos do trabalho. Na literatura, como ressaltado por Fields (2003), o mesmo termo "mobilidade econômica" (ou mobilidade social) é utilizado para eventos com significados diferentes e diversas interpretações podem ser dadas para sua definição.

A quantificação da variação observada nos rendimentos médios dos indivíduos, derivada por Fields e Ok (1996), denominada mobilidade direcional de rendimentos (mdr), é expressa por:

$$
m d r(x, y)=\frac{1}{n} \sum_{i=1}^{n}\left(\log x_{i}-\log y_{i}\right)
$$

em que $x_{i}$ e $y_{i}$ representam, respectivamente, o rendimento do indivíduo $i$ no período final e no inicial. Esta medida combina as perdas e ganhos dos rendimentos ao passo que o efeito das trocas entre os indivíduos é considerado pelo indicador movimento não direcional dos rendimentos (mndr), que é definido como:

$$
m n d r(x, y)=\frac{1}{n} \sum_{i=1}^{n}\left|\log x_{i}-\log y_{i}\right|
$$

A decomposição deste indicador torna possível mensurar o efeito da mobilidade em dois aspectos: efeito distributivo, no qual a mobilidade pode resultar de uma possível alteração na posição dos indivíduos dentro da distribuição de rendimentos e, efeito desempenho econômico, que reflete a relação da mobilidade com períodos de crescimento ou estagnação da economia. Esta é dada por: 


$$
m n d r(x, y)=|\underline{\underline{m d r(x, y)}}|+\underline{\underline{((m n d r)-|m d r(x, y)|)}}
$$

em que a primeira parcela é explicada pelo crescimento e a segunda, por possíveis mudanças de posição na distribuição.

Somando-se a estes indicadores, tem-se a análise da dependência temporal e do movimento de participação. A primeira indica quanto os rendimentos presentes estão, ou não, correlacionados com os passados. Para isso, são utilizados os indicadores tradicionais de mobilidade, além das matrizes de mobilidade quantílica. Estas matrizes classificam os indivíduos, em cada período, de acordo com quantis, que são, no ano-base, a linha da matriz, e, no período final, a coluna da mesma.

O movimento de participação (mpr), por sua vez, permite mensurar a participação de cada indivíduo em relação à média dos rendimentos totais sendo calculado como segue:

$$
m p r(x, y)=\frac{\sum_{i=1}^{n}\left|\frac{x_{i}}{\bar{x}}-\frac{y_{i}}{\bar{y}}\right|}{n}
$$

em que $\bar{x}$ e $\bar{y}$ representam, respectivamente, a média dos rendimentos totais no período final e no período inicial e $\mathrm{n}$, o total de indivíduos da amostra.

Por fim, uma ênfase no papel da mobilidade como instrumento equalizador de rendimentos de longo prazo é considerada. Os índices de Fields (2005), Chakravarty, Dutta e Weimark (1985) e Shorrock's (1978) podem ser considerados. Contudo, quando o objetivo é medir as consequências de bem-estar da mobilidade de renda relativa, o índice de Fields (2005) é o mais adequado. Este índice compara o bem-estar entre a distribuição de renda atual e a distribuição no ano-base, e, não relativa a um caminho hipotético como o fazem os outros autores supracitados.

No índice de Fields, a mobilidade em direção à equalização depende da relação entre a desigualdade da renda média e a desigualdade de renda inicial. Se a renda média é distribuída mais (ou menos) igualmente que a renda inicial, a mobilidade tende a equalizar (ou desequalizar) a renda de longo prazo relativa à renda de referência. O índice é expresso por:

$$
E \equiv 1-\left(I(a) / I\left({ }_{y} 1\right)\right)
$$

em que $a$ é o vetor de rendimentos médios, ${ }_{y} 1$ é o vetor de rendimentos no ano-base e $I($.$) é a medida de desigualdade. Quando os rendimentos são distribuídos,$ ao longo do tempo, mais desigualmente com relação ao período-base, $\mathrm{E}<0$, e, quando são distribuídos mais igualmente, $\mathrm{E}>0$. 


\section{MOBILIDADE DE RENDIMENTOS A PARTIR DE DADOS INDIVIDUAIS}

A análise dos indicadores de mobilidade por meio dos dados individuais da PME é centrada nas seis regiões metropolitanas que constituem a pesquisa e é subdividida em dois períodos, 1992-2001 e 2002-2009¹.

\section{Período 1992-2001}

O indicador de mobilidade direcional mostra uma variação positiva mais significativa dos ganhos médios individuais apenas no período de 1994-1995. A recuperação dos rendimentos neste período pode ser atribuída ao sucesso do Plano Real no combate à inflação, com expressivo aumento da renda real dos trabalhadores. Os resultados ínfimos observados a partir deste período podem ter origem em fatores conjunturais que atingiram a economia brasileira. A ocorrência das crises asiática e russa, em 1997 e 1998, respectivamente, e a crise cambial brasileira, em 1999, afetaram diretamente os rendimentos dos trabalhadores brasileiros no período. A maior variação negativa dos rendimentos médios individuais é registrada em 1998-1999 ficando, assim, evidente o impacto das crises, internacionais e brasileira, na renda dos trabalhadores.

A mobilidade não direcional também é mais expressiva no biênio 1994-1995, quando passa a apresentar tendência decrescente. Os fatores conjunturais que interferiram na economia brasileira ao longo do período são evidenciados pela decomposição deste indicador nos efeitos desempenho e distributivo. O de desempenho, dado pelo módulo do indicador de mobilidade direcional, tem seu maior patamar no período 1994-1995, acompanhado pelo período 2000-2001. Nestes dois biênios, o efeito distributivo atinge seus menores valores.

O movimento de participação apresenta tendência decrescente no período, com recuperação a partir de 1998-1999. Em 1992-1993, a participação individual aumenta em 0,56 enquanto em 1996-1997 atinge seu menor patamar, com um nível de 0,44. Este indicador encontra-se em patamar mais elevado do que os EUA e a França como reportam Fields et al. (2000) e Buchinsky et al. (2003), para os EUA (entre 1970 e 1995) e para a França (a partir de 1978), respectivamente.

De forma geral, a mobilidade de rendimentos pode ser sentida em todo o período, ainda que com tendência decrescente ao longo do tempo, voltando a ampliar no último biênio. Pelo efeito distributivo e pelo movimento de participação, houve uma troca dos indivíduos na distribuição, mas não se pode dizer quem foi mais ou menos beneficiado. As direções de mobilidade, mobilidade ascendente, imobilidade e mobilidade descendente, e matrizes de transição elucidam este comportamento.

A imobilidade aumenta ao longo do período e a mobilidade ascendente registrada é menor que a descendente. A distribuição das frequências das matrizes de transição mostram que a menor imobilidade nos dois primeiros biênios age no

\footnotetext{
${ }^{1}$ A descrição da base de dados e os indicadores de mobilidade estão nos Anexos.
} 
sentido de uma maior equalização dos rendimentos individuais. Nestes dois períodos, com destaque para o primeiro, a mobilidade ascendente é mais expressiva. Ao mesmo tempo, os $20 \%$ mais ricos concentram a maior mobilidade descendente. A partir de 1996, os 20\% mais ricos registram um aumento da imobilidade.

Os indicadores desagregados pelas características individuais e do mercado de trabalho registram as mesmas tendências observadas para os indicadores em termos totais ${ }^{2}$. Eles não se diferenciam para sexo e posição no domicílio. Sem controlar por outros fatores, o nível de escolaridade dos indivíduos pode justificar uma maior mobilidade para aqueles com menor nível de instrução. Os rendimentos daqueles com 12 anos ou mais de estudo respondem, de modo geral, de forma mais expressiva ao comportamento conjuntural que os demais. Um padrão homogêneo não é evidente ao se considerar o efeito distributivo. O efeito distributivo dos menos escolarizados é bem próximo dos achados para os mais escolarizados. Entretanto, pelo movimento de participação, os menos escolarizados apresentam indicadores mais elevados.

O padrão U-invertido observado para a participação no mercado de trabalho é refletido na remuneração dos trabalhadores. Com o tempo, o aumento da experiência e da escolaridade tende a se traduzir em maiores rendimentos, acarretando, assim, maior mobilidade. A maior mobilidade para as gerações mais jovens pode ser justificada pelo maior nível educacional destas em relação às coortes mais antigas. O efeito da mudança composicional educacional para as gerações mais jovens pode explicar, assim, a melhora na distribuição de rendimentos destas gerações em detrimento das demais. A elevada mobilidade para as coortes mais novas é confirmada por autores como Gutierrez (2004) para a Argentina, Nascimento e Souza (2005) para o Brasil e, ainda, por Finnie (1997) para o Canadá, Bigard et al. (1998) para os casos francês e italiano e Wodon (2001) para Argentina e México.

Quando levamos em conta características referentes ao mercado de trabalho, pelo movimento de participação, os resultados destacam aqueles pertencentes ao setor informal e que apresentam jornada de trabalho parcial. Mas o aumento no rendimento médio destes indivíduos é proveniente de fatores conjunturais e não de uma melhora distributiva. Por fim, aqueles residentes nas RMs de Recife, Salvador e Belo Horizonte registram indicadores mais acentuados para o efeito distributivo e movimento de participação.

\section{Período 2002-2009}

A mobilidade direcional se mantém bem menos expressiva que a mobilidade não direcional entre 2002 e 2009 . E, pela decomposição da mobilidade não direcional, fica evidente a origem da mobilidade, dada a maior expressão do efeito distributivo. Este comportamento é confirmado pelo movimento de participação,

\footnotetext{
${ }^{2}$ Os resultados para estes indicadores de mobilidade estão disponíveis para quem os solicitar.
} 
que ganha destaque pela sua maior magnitude entre os indicadores considerados. Tais resultados seguem o mesmo comportamento observado para o período 1992-2001. Pela mobilidade direcional, o único período que caracteriza uma perda média dos rendimentos é 2002-2003. A partir de 2003-2004, uma tendência oposta e crescente pode ser observada até o ano de 2006, quando este indicador atinge seu valor máximo de 0,0538 . A partir deste ano, os ganhos médios individuais sofrem uma redução.

O efeito desempenho, ainda que tenha sido mais expressivo em 2002-2003, mostra que os fatores conjunturais da economia afetaram a mobilidade de uma forma negativa. A importância do efeito distributivo sobre o efeito econômico é registrada para todo o período. Os anos de 2002 e 2003 registram a maior troca de posição dos indivíduos, com tendência decrescente até o ano de 2006, quando atinge seu menor patamar, voltando a se elevar a partir de então. Este resultado é confirmado pelo movimento de participação.

As direções de mobilidade reiteram os demais indicadores. Uma maior imobilidade dos rendimentos é sentida ao longo do tempo, revertendo-se no último período. A imobilidade no período 2002-2003 é de $45 \%$, chegando a atingir $55 \%$ em 2006-2007. A mobilidade descendente ocorre em maior patamar do que a ascendente para todo o período. Pela distribuição das frequências das matrizes de transição, pode-se notar que o movimento de ascensão é maior para os mais pobres nos primeiros biênios.

\section{CONSIDERAÇÕES FINAIS}

A análise da mobilidade em um contexto intrageracional foi possível com os dados individuais da PME. No primeiro período (1992-2002), os indicadores prevalecem sobre os do período atual (2002-2009). Ainda que o movimento de participação e o efeito distributivo apresentem redução ao longo dos dois períodos, eles confirmam uma maior mobilidade ascendente para os mais pobres quando consideradas as matrizes de transição. Não são observadas diferenças quanto ao sexo, posição no domicílio e, no período mais recente, entre brancos e não brancos. Um comportamento positivo para a mobilidade é observado para indivíduos com menor nível de escolaridade, nascidos em coortes mais recentes, inseridos no setor informal e com jornada de trabalho integral. E, ainda, uma melhora na distribuição de rendimentos é apontada para os indivíduos residentes nas RMs de Belo Horizonte, Recife e Salvador.

Em suma, este atigo enfatizou fatos estilizados sobre a mobilidade de rendimentos no Brasil por meio de dados longitudinais. Em vista dos resultados encontrados, percebe-se uma relação entre a mobilidade e a queda da desigualdade. A mobilidade contribui para desconcentração de renda e, se persistir, pode levar, no longo prazo, a uma maior equalização dos rendimentos. 


\section{REFERÊNCIAS BIBLIOGRÁFICAS}

ANDRADE, F.C.D. (2000) A evolução da mobilidade social em cinco regiões metropolitanas brasileiras, 1988 e 1996". In: ENCONTRO NACIONAL DE ESTUDO POPULACIONAIS, 12, 2000, Caxambu, Anais. Caxambu: ABEP. (CD-ROM)

ANDRADE, E. et al. (2003) "Do borrowing constraints decrease intergenerational mobility in Brazil? A test using quantile regression. [S.1].

AYDEMIR, Abdurrahman; CHEN, Wen-Hao; CORAK, Miles. (2005) "Intergenerational earnings mobility among the children of Canadian immigrants". Analytical Studies Branch Research Paper Series 2005267, Statistics Canada, Analytical Studies Branch.

BARROS, R.P. ; FOGUEL, M.N.; ULYSSEA, G. (2006) "Sobre a recente queda da desigualdade de renda no Brasil”. In: BARROS, R.P. ; FOGUEL, M.N.; ULYSSEA, G. (Orgs.) Desigualdade de renda no Brasil: uma análise da queda recente. Brasília: IPEA, v. 1.

BEACH, C. M.; FINNIE, R. (2004) "A longitudinal analysis of earnings change in Canada", Canadian Journal of Economics, v.37, n. 1, February.

BECKER, G. S.; TOMES, N. (1979) "An equilibrium theory of the distribution of income and intergenerational mobility", Journal of Political Economy, 87, p. 1153-1189.

BECKER, G. S.; TOMES, N. (1986) "Human capital and the rise and fall of families", Journal of Labor Economics, 4, p. 1-39.

BEHRMAN, J. R.; GAVIERIA, A. U.; SZEKELY, M. S. (2001) "Intergenerational Mobility in Latin America. Inter-American Development Bank, Research Department Working Papers Series, n. 452.

BIGARD, A.; GUILLOTIN, Y.; LUCIFORA, C. (1998) "Earnings mobility: an international comparison of Italy and France", Review of Income and Wealth, série 44, n. 4, p. 473-495.

BJÖRKLUND, A.; JÄNTTI, M. (2000) "Intergenerational mobility of socio-economic status in comparative perspective", Nordic Journal of Political Economy, 26, p. 3-32.

BUCHINSKY, Moshe; FIELDS, Gary; FOUGÈRE, Denis; KRAMARZ, Francis. (2003) "Francs or Ranks? Earnings Mobility in France, 1967-1999”. London: Centre for Economic Policy Research, C.E.P. R. Discussion Papers, n. 3937, June 2003.

CHAKRAVARTY, S.J.; DUTTA, B.; WEYMARK, J.A. (1985) "Ethical indices of income mobility", Social Choice and Welfare, 2, p. 1-21.

CORAK, M.; HEISZ, A. (1999) "The intergenerational earnings and income mobility of Canadian men: evidence from longitudinal income tax”, Business and Labour Market Análisis, n. 113.

CORSEUIL, C.H.; FOGUEL, M.N. (2002) "Uma sugestão de deflatores para rendas obtidas a partir de algumas pesquisas domiciliares do IBGE”, Rio de Janeiro: IPEA, Texto para Discussão n. 897.

ERMISCH J.; NICOLETTI, C. (2005) "Intergenerational earnings mobility: Changes across cohorts in Britain”, ISER Working Paper 2005-19. Colchester: University of Essex.

ERIKSON, R.; GOLDTHORPE, J.H. (2002) “Intergenerational inequality: a sociological perspective", Journal of Economic Perspective, 16, p. 31-44.

ERMISCH, J.; FRANCESCONI, J. (2004) "Intergenerational mobility in Britain: new evidence from the British household panel study”. In: CORAK, M. (Ed.) Generational Income Mobility in North America and Europe. New York: Cambridge University Press.

FERREIRA, S.G.; VELOSO, F.A. (2004) "Intergenerational Mobility of wages in Brazil”. Meeting of the Network in inequality and poverty (NIP). November 2004.

FIELDS, Gary; LEARY, Jesse; OK, Efe. (2000) "Dollars and deciles: changing earnings mobility in the United States, 1970-1995", Cornell University Working Paper.

FIELDS, Gary; OK, Efe. (1996) "The meaning and measurement of income mobility", Journal of Economic Theory. n. 71, p. 349-377.

FIELDS, Gary. (2005) "Does income mobility equalize longer-term incomes? New measures of an old concept”. Paper presented on Frontiers on Social and Economic Mobility Conference. New York: Cornell University, July.

FIGUEIREDO, Erick A. (2010) “Mobilidade intrageracional de renda no Brasil”, Nova Economia, vol. $20, \mathrm{n} \cdot 3$.

FINNIE, Ross. (1997) “Earnings mobility in Canada”, Journal of Income Distribution, p. 77-107. 
GOLDTHORPE, J.; ERICKSON, R. (1993) The constant flux: a study of class mobility in industrial societies. Oxford: Oxford University Press.

GUTIERREZ, F. (2004) "Dinàmica salarial y ocupacional: anàlisis de panel para Argentina: 19982002”, CEDLAS, Universidad Nacional de La Plata, Working Paper n. 11.

IBGE. Série histórica da pesquisa mensal de emprego. Disponível em <www.ibge.gov.br>.

IBGE. Série histórica da pesquisa nacional por amostra de domicílios. Disponível em <www.ibge.gov.br>.

MACHADO, A. F.; RIBAS, Rafael Perez. (2008) "Mudanças no mercado de trabalho retiram famílias da pobreza? Determinantes domiciliares e agregados para a saída da pobreza nas regiões metropolitanas do Brasil”. Texto para Discussão IPEA, Brasília: IPEA, 2008.

NASCIMENTO, Marcos Aurélio; SOUZA, André Portela. (2005) “Medidas e determinantes dos rendimentos do trabalho no Brasil". Encontro Nacional de Economia, Natal, 2005

PASTORE, J. (1979) Desigualdade e mobilidade social no Brasil. São Paulo: T.A.Queiroz. Ed. da Universidade de São Paulo.

PASTORE, J.; HALLER, A. (1993) “O que está acontecendo com a mobilidade social no Brasil?” In Albuquerque, R.; Velloso, J.P. R. (Orgs.). Pobreza e mobilidade social. São Paulo: Nobel, p. 25-52.

PASTORE, J.; VALLE SILVA, N. (2000) Mobilidade social no Brasil. São Paulo: Makron.

PERO, V.; SZERMAN, D. (2008) “Mobilidade intergeracional de renda no Brasil.”, Pesquisa e Planejamento Econômico, Rio de Janeiro, v. 38, n. 1, p. 1-35.

SHORROCKS, A.F. (1978) “The measurement of mobility”, Econometrica, v.46, n. 5, p. 1013-24.

SOARES, S.; OSORIO, R.G.; SOARES, F.V.; MEDEIROS, M.; ZEPEDA, E. (2007) "Conditional cash transfer in Brazil, Chile and México: impacts upon inequality” Brasília, DF: International Poverty Center, working paper n. 35, April, 2007.

SOLON, G. (2001) "Mobility within and between Generations”, in Finis Welch (ed.), The Causes and Consequences of Increasing Inequality, p. 153-168, Chicago: University of Chicago Press.

WODON, Q. (2001) "Income mobility and risk during the business cycle: comparing adjustments in labor market in two Latin American Countries”, Economics of Transition, 9, p. 449-461.

\section{ANEXOS}

\section{A1. Fonte de dados}

A PME é uma pesquisa organizada em forma longitudinal, ainda que sob formato de painel rotativo. As informações individuais reportadas na PME permitem mensurar a mobilidade em um contexto intrageracional. Contudo, esta pesquisa apresenta uma quebra temporal em 2002, quando o IBGE mudou a metodologia, em especial, o conceito de trabalho. Desse modo, a mobilidade é mensurada nos intervalos de 1992 a 2002 e 2002 a 2009, nas seis regiões metropolitanas — Recife, Salvador, Belo Horizonte, São Paulo, Rio de Janeiro e Porto Alegre - que compõem a pesquisa. O painel rotativo permite acompanhar os domicílios durante quatro meses consecutivos e, após um intervalo de oito meses, eles voltam a ser investigados por mais quatro meses, quando são definitivamente excluídos da amostra. Este esquema de rotação garante o caráter longitudinal da pesquisa.

A amostra é formada por trabalhadores, com idade entre 26 e 65 anos, que apresentaram rendimento-hora ${ }^{3}$ do trabalho positivo na $1^{\text {a }}$ e na $5^{\text {a }}$ quinta entrevista e aqueles que, na primeira entrevista, estavam desempregados e inativos, mas que tiveram

\footnotetext{
${ }^{3}$ A variável de rendimentos foi deflacionada pelo deflator para rendimentos da PME proposto por Corseuil e Foguel (2002).
} 
renda do trabalho positiva na quinta entrevista. Para o primeiro subperíodo, a análise é realizada considerando biênios ${ }^{4}$, onde o primeiro período corresponde à $1^{\mathrm{a}}$ entrevista e o segundo à $5^{\text {a }}$ entrevista.

Ainda, com o objetivo de mensurar o efeito de características do mercado de trabalho sobre a mobilidade, consideram-se as seguintes variáveis: posição na ocupação em que o trabalhador se encontra: o setor formal é definido pelo indivíduo com carteira de trabalho assinada, empregador, servidor público e profissional liberal, e o setor informal fica, assim, composto pelo trabalhador sem carteira de trabalho assinada e pelo trabalhador por conta própria sem os profissionais liberais; e jornada de trabalho: integral e parcial, sendo a integral formada por mais de 30 horas de trabalho semanal.

No primeiro período considerado, 1992-2001, a amostra conta com maioria de homens e chefes de domicílio, que têm sua proporção reduzida ao longo do período. Houve um aumento da proporção de trabalhadores mais escolarizados (com 11 anos ou mais de estudo) e uma redução mais significativa daqueles com até 3 anos de estudo. Quanto às características do mercado de trabalho, predominam aqueles inseridos no setor formal e com jornada de trabalho integral e residentes nas RM's de São Paulo e Belo Horizonte.

Entre 2002 e 2009, a amostra é composta de uma maioria de homens, brancos ${ }^{5}$ e chefes de domicílio, com redução na proporção de 2002 a 2009. Indivíduos com 4 a 7 anos de estudo e com 11 anos completos de estudo são a maioria. Quanto às características relativas ao mercado de trabalho, predominam indivíduos no setor formal, com jornada de trabalho integral (acima de 30 horas) e que contribuem para a previdência. Ainda, residentes nas regiões metropolitanas de São Paulo e Rio de Janeiro constituem maior parte da amostra.

\section{A2. Indicadores de Mobilidade}

\begin{tabular}{|c|c|c|c|c|c|}
\hline \multicolumn{6}{|c|}{$\begin{array}{l}\text { Tabela A1: Indicadores de mobilidade: rendimento-hora } \\
\text { do trabalho principal, Brasil, 1992-2001 }\end{array}$} \\
\hline & $1992-1993$ & 1994-1995 & 1996-1997 & 1998-1999 & 2000-2001 \\
\hline Mobilidade direcional & 0.0234 & 0.2115 & 0.0977 & 0.0514 & 0.1079 \\
\hline Mobilidade não direcional & 0.5420 & 0.5765 & 0.4936 & 0.4900 & 0.4908 \\
\hline Efeito desempenho & 0.0234 & 0.2115 & 0.0977 & 0.0514 & 0.1079 \\
\hline Efeito distributivo & 0.5186 & 0.3650 & 0.3959 & 0.4387 & 0.3830 \\
\hline Movimento de participação & 0.5561 & 0.5480 & 0.4391 & 0.4445 & 0.4438 \\
\hline
\end{tabular}

Fonte: PME, 1992-2001.

\footnotetext{
${ }^{4} \mathrm{Na}$ PME antiga o processo de rotação do painel leva à substituição de $100 \%$ da amostra a cada par de anos enquanto na nova PME, a cada ano, metade da amostra é sempre comum.

${ }^{5}$ Consideram-se brancos (brancos e amarelos) e não brancos (pretos, pardos e indígenas).
} 
Tabela A2: Mobilidade Ascendente, Imobilidade e Mobilidade Descendente: rendimento-hora do trabalho principal, Brasil, 1992-2001

\begin{tabular}{cccc}
\hline Ano & Mobilidade Ascendente (\%) & Imobilidade (\%) & Mobilidade Descendente (\%) \\
\hline $1992-1993$ & 28.21 & 35.17 & 36.62 \\
$1994-1995$ & 26.42 & 40.04 & 33.54 \\
$1996-1997$ & 24.70 & 46.52 & 28.79 \\
$1998-1999$ & 24.83 & 46.22 & 28.95 \\
$2000-2001$ & 24.24 & 46.60 & 29.17 \\
\hline
\end{tabular}

Fonte: PME, 1992-2001.

Tabela A3: Distribuição das frequências da matriz de transição: rendimento-hora do trabalho principal, Brasil Metropolitano, 1992-2001

\begin{tabular}{ccccccc}
\hline \multicolumn{7}{c}{$1992-1993$} \\
\hline \multirow{4}{*}{$1^{\circ}$ entrevista } & $1^{\circ}$ & $2^{\circ}$ & $3^{\circ}$ & $4^{\text {o }}$ & $5^{\text {o }}$ \\
\hline & $1^{\circ}$ & 8.72 & 4.49 & 3.08 & 2.36 & 1.35 \\
& $2^{\circ}$ & 6.84 & 6.28 & 4.00 & 1.95 & 0.94 \\
& $3^{\text {o }}$ & 2.70 & 5.25 & 5.85 & 4.50 & 1.70 \\
& $4^{\circ}$ & 1.20 & 2.95 & 4.92 & 6.40 & 4.53 \\
& $5^{\circ}$ & 0.53 & 1.03 & 2.17 & 4.80 & 11.48 \\
\hline
\end{tabular}

\begin{tabular}{ccccccc}
\hline \multicolumn{7}{c}{ 1994-1995 } \\
\hline \multirow{5}{*}{$1^{\circ}$ entrevista } & $1^{\circ}$ & $2^{\circ}$ & $3^{\circ}$ & $4^{\text {o }}$ & $5^{\text {o }}$ \\
\hline & $1^{\text {o }}$ & 9.16 & 4.76 & 3.14 & 2.00 & 0.94 \\
& $2^{\text {o }}$ & 7.12 & 6.58 & 4.01 & 1.82 & 0.47 \\
& $3^{\text {o }}$ & 2.69 & 5.71 & 6.25 & 4.25 & 1.10 \\
& $4^{\text {o }}$ & 0.84 & 2.49 & 5.26 & 7.58 & 3.83 \\
& $5^{\text {o }}$ & 0.20 & 0.45 & 1.34 & 4.35 & 13.66 \\
\hline
\end{tabular}

\begin{tabular}{ccccccc}
\multicolumn{7}{c}{$1996-1997$} \\
\hline \multirow{5}{*}{$1^{\circ}$ entrevista } & $1^{\circ}$ & $2^{\circ}$ & $3^{\circ}$ & $4^{\circ}$ & $5^{\circ}$ \\
\hline & $1^{\circ}$ & 10.48 & 4.61 & 2.49 & 1.59 & 0.83 \\
& $2^{\circ}$ & 6.45 & 7.96 & 3.83 & 1.50 & 0.27 \\
& $3^{\circ}$ & 2.12 & 5.16 & 7.94 & 3.94 & 0.84 \\
& $4^{\circ}$ & 0.81 & 1.82 & 4.72 & 9.28 & 3.37 \\
& $5^{\circ}$ & 0.13 & 0.45 & 1.02 & 3.70 & 14.70 \\
\hline
\end{tabular}

\begin{tabular}{ccccccc}
\multicolumn{7}{c}{ 1998-1999 } \\
\hline \multirow{5}{*}{$1^{\circ}$ entrevista } & $1^{\circ}$ & $2^{\circ}$ & $3^{\circ}$ & $4^{\circ}$ & $5^{\circ}$ \\
\hline & $1^{\circ}$ & 10.16 & 4.64 & 2.68 & 1.72 & 0.79 \\
& $2^{\circ}$ & 6.62 & 8.13 & 3.68 & 1.26 & 0.31 \\
& $3^{\text {o }}$ & 2.22 & 4.99 & 8.04 & 3.99 & 0.77 \\
& $4^{\text {o }}$ & 0.81 & 1.89 & 4.54 & 9.57 & 3.20 \\
& $5^{\text {o }}$ & 0.19 & 0.35 & 1.06 & 3.47 & 14.93 \\
\hline
\end{tabular}

\begin{tabular}{ccccccc}
\multicolumn{7}{c}{$\mathbf{2 0 0 0 - 2 0 0 1}$} \\
\hline \multirow{6}{*}{$1^{\circ}$ entrevista } & $1^{\text {o }}$ & $2^{\text {o }}$ entrevista & $3^{\text {o }}$ & $4^{\text {o }}$ & $5^{\text {o }}$ \\
\hline & $1^{\text {o }}$ & 10.02 & 4.45 & 2.93 & 1.66 & 0.95 \\
& $2^{\text {o }}$ & 6.81 & 8.04 & 3.50 & 1.34 & 0.31 \\
& $3^{\text {o }}$ & 2.16 & 5.40 & 7.72 & 3.81 & 0.91 \\
& $4^{\text {o }}$ & 0.84 & 1.75 & 4.80 & 9.40 & 3.21 \\
& $5^{\text {o }}$ & 0.18 & 0.37 & 1.05 & 3.79 & 14.62 \\
\hline
\end{tabular}

Fonte: PME, 1992-2001.

Tabela A4: Indicadores de mobilidade: rendimento-hora do trabalho principal, Brasil, 2002-2009

\begin{tabular}{lccccccc}
\hline & $2002-03$ & $2003-04$ & $2004-05$ & $2005-06$ & $2006-07$ & $2007-08$ & $2008-09$ \\
\hline Mobilidade direcional & -0.0715 & 0.0128 & 0.0382 & 0.0541 & 0.0538 & 0.0419 & 0.0283 \\
$\begin{array}{l}\text { Mobilidade } \\
\text { não direcional }\end{array}$ & 0.4222 & 0.3548 & 0.3372 & 0.3326 & 0.3133 & 0.3196 & 0.3202 \\
$\begin{array}{l}\text { Efeito desempenho } \\
\text { Efeito distributivo }\end{array}$ & 0.0715 & 0.0128 & 0.0382 & 0.0541 & 0.0538 & 0.0419 & 0.0283 \\
$\begin{array}{l}\text { Movimento } \\
\text { de participação }\end{array}$ & 0.3507 & 0.3420 & 0.2990 & 0.2785 & 0.2595 & 0.2777 & 0.2920 \\
\hline
\end{tabular}

Fonte: PME, 2002-2009. 
Tabela A5: Mobilidade Ascendente, Imobilidade e Mobilidade Descendente: rendimento-hora do trabalho principal, Brasil, 2002-2009

\begin{tabular}{cccc}
\hline Ano & Mobilidade Ascendente (\%) & Imobilidade (\%) & Mobilidade Descendente (\%) \\
\hline $2002-03$ & 24.41 & 45.04 & 30.55 \\
$2003-04$ & 21.77 & 50.03 & 28.21 \\
$2004-05$ & 21.54 & 51.99 & 26.47 \\
$2005-06$ & 21.75 & 53.00 & 25.25 \\
$2006-07$ & 19.89 & 54.79 & 25.32 \\
$2007-08$ & 20.82 & 53.58 & 25.59 \\
$2008-09$ & 21.33 & 52.78 & 25.89 \\
\hline
\end{tabular}

Fonte: PME, 2002-2009.

Tabela A6: Distribuição das frequências da matriz de transição: rendimento-hora do trabalho principal, Brasil Metropolitano, 2002-2009

\begin{tabular}{|c|c|c|c|c|c|c|}
\hline \multicolumn{7}{|c|}{$2002-2003$} \\
\hline \multicolumn{7}{|c|}{$5^{\circ}$ entrevista } \\
\hline & & $1^{\circ}$ & $2^{\circ}$ & $3^{\circ}$ & $4^{\circ}$ & $5^{\circ}$ \\
\hline \multirow{5}{*}{$1^{\circ}$ entrevista } & $1^{\circ}$ & 5.13 & 2.92 & 1.58 & 0.80 & 0.22 \\
\hline & $2^{\circ}$ & 2.97 & 5.71 & 4.15 & 1.90 & 0.45 \\
\hline & $3^{\circ}$ & 1.58 & 3.87 & 8.54 & 5.11 & 1.09 \\
\hline & $4^{\circ}$ & 0.99 & 1.92 & 4.87 & 11.49 & 4.84 \\
\hline & $5^{\circ}$ & 0.26 & 0.55 & 1.24 & 4.47 & 23.36 \\
\hline \multicolumn{7}{|c|}{ 2004-2005 } \\
\hline \multicolumn{7}{|c|}{$5^{\circ}$ entrevista } \\
\hline & & $1^{\circ}$ & $2^{\circ}$ & $3^{\circ}$ & $4^{\circ}$ & $5^{\circ}$ \\
\hline \multirow{5}{*}{$1^{\circ}$ entrevista } & $1^{\circ}$ & 7.12 & 3.53 & 1.48 & 0.65 & 0.20 \\
\hline & $2^{\circ}$ & 3.05 & 7.22 & 3.72 & 1.47 & 0.33 \\
\hline & $3^{\circ}$ & 1.53 & 3.78 & 9.43 & 4.21 & 0.71 \\
\hline & $4^{\circ}$ & 0.68 & 1.50 & 4.36 & 12.91 & 3.81 \\
\hline & $5^{\circ}$ & 0.16 & 0.31 & 0.81 & 3.94 & 23.06 \\
\hline \multicolumn{7}{|c|}{ 2006-2007 } \\
\hline \multicolumn{7}{|c|}{$5^{\circ}$ entrevista } \\
\hline & & $1^{\circ}$ & $2^{\circ}$ & $3^{\circ}$ & $4^{\circ}$ & $5^{\circ}$ \\
\hline \multirow{5}{*}{$1^{\circ}$ entrevista } & $1^{\circ}$ & 7.58 & 3.09 & 1.43 & 0.64 & 0.15 \\
\hline & $2^{\circ}$ & 3.32 & 7.41 & 3.48 & 1.34 & 0.33 \\
\hline & $3^{\circ}$ & 1.25 & 4.32 & 9.51 & 3.97 & 0.83 \\
\hline & $4^{\circ}$ & 0.65 & 1.20 & 4.63 & 12.71 & 3.57 \\
\hline & $5^{\circ}$ & 0.17 & 0.31 & 0.82 & 4.03 & 23.24 \\
\hline \multicolumn{7}{|c|}{ 2008-2009 } \\
\hline \multicolumn{7}{|c|}{$5^{\circ}$ entrevista } \\
\hline & & $1^{\circ}$ & $2^{\circ}$ & $3^{\circ}$ & $4^{\circ}$ & $5^{\circ}$ \\
\hline \multirow{5}{*}{$1^{\circ}$ entrevista } & $1^{\circ}$ & 7.48 & 3.12 & 1.56 & 0.72 & 0.18 \\
\hline & $2^{\circ}$ & 3.74 & 7.30 & 3.70 & 1.43 & 0.36 \\
\hline & $3^{\circ}$ & 1.43 & 4.58 & 8.98 & 3.94 & 0.78 \\
\hline & $4^{\circ}$ & 0.74 & 1.58 & 4.32 & 12.30 & 3.81 \\
\hline & $5^{\circ}$ & 0.26 & 0.35 & 1.06 & 3.81 & 22.45 \\
\hline
\end{tabular}

\begin{tabular}{|c|c|c|c|c|c|c|}
\hline \multicolumn{7}{|c|}{ 2003-2004 } \\
\hline \multicolumn{7}{|c|}{$5^{\circ}$ entrevista } \\
\hline & & $1^{\circ}$ & $2^{\circ}$ & $3^{\circ}$ & $4^{\circ}$ & $5^{\circ}$ \\
\hline \multirow{5}{*}{$1^{\circ}$ entrevista } & $1^{\circ}$ & 6.58 & 3.18 & 1.46 & 0.74 & 0.22 \\
\hline & $2^{\circ}$ & 3.24 & 6.63 & 4.13 & 1.62 & 0.33 \\
\hline & $3^{\circ}$ & 1.42 & 3.76 & 9.41 & 4.76 & 0.93 \\
\hline & $4^{\circ}$ & 0.82 & 1.55 & 4.89 & 12.64 & 3.99 \\
\hline & $5^{\circ}$ & 0.23 & 0.33 & 0.97 & 3.91 & 22.26 \\
\hline \multicolumn{7}{|c|}{ 2005-2006 } \\
\hline \multicolumn{7}{|c|}{$5^{\circ}$ entrevista } \\
\hline & & $1^{\circ}$ & $2^{\circ}$ & $3^{\circ}$ & $4^{\circ}$ & $5^{\circ}$ \\
\hline \multirow{5}{*}{$1^{\circ}$ entrevista } & $1^{\circ}$ & 7.13 & 2.99 & 1.48 & 0.66 & 0.15 \\
\hline & $2^{\circ}$ & 3.03 & 6.99 & 3.96 & 1.53 & 0.25 \\
\hline & $3^{\circ}$ & 1.45 & 3.86 & 9.43 & 4.38 & 0.98 \\
\hline & $4^{\circ}$ & 0.70 & 1.30 & 4.50 & 12.87 & 3.82 \\
\hline & $5^{\circ}$ & 0.23 & 0.29 & 0.77 & 4.10 & 23.16 \\
\hline \multicolumn{7}{|c|}{ 2007-2008 } \\
\hline \multicolumn{7}{|c|}{$5^{\circ}$ entrevista } \\
\hline & & $1^{\circ}$ & $2^{\circ}$ & $3^{\circ}$ & $4^{\circ}$ & $5^{\circ}$ \\
\hline \multirow{5}{*}{$1^{\circ}$ entrevista } & $1^{\circ}$ & 7.48 & 3.29 & 1.41 & 0.74 & 0.22 \\
\hline & $2^{\circ}$ & 3.39 & 7.43 & 3.49 & 1.30 & 0.31 \\
\hline & $3^{\circ}$ & 1.54 & 4.26 & 9.67 & 4.00 & 0.92 \\
\hline & $4^{\circ}$ & 0.70 & 1.34 & 4.61 & 12.60 & 3.65 \\
\hline & $5^{\circ}$ & 0.20 & 0.37 & 0.89 & 3.89 & 22.32 \\
\hline
\end{tabular}

Fonte: PME, 2002-2009. 